\title{
El Máster de Formación Inicial del Profesorado de Secundaria y el conocimiento práctico profesional del futuro profesorado de Ciencias Experimentales, Matemáticas y Tecnología
}

\author{
Jenaro Guisasola ${ }^{1}$, José Ignacio Barragués ${ }^{2}$, Mikel Garmendia ${ }^{3}$ \\ ${ }^{1}$ Miembro de la Comisión Académica del MFPS, Universidad del País V asco-Euskal Herriko \\ Unibertsitatea, Donostia-San Sebastián.España.jenaro.guisasola@ebu.es \\ ${ }^{2}$ Coordinador del módulo especifico de CCNN y Matemáticas del MFPS, Universidad del País V asco- \\ Euskal Herriko Unibertsitatea, Donostia-San Sebastián.España.barragues@gmail.com \\ ${ }^{3}$ Coordinador del módulo especifico de Tecnologia del MFPS, Universidad del País V asco-Euskal Herriko \\ Unibertsitatea, Donostia-San Sebastian.España.garmendia@gmail.com
}

[Recibido en septiembre de 2012, aceptado en enero de 2013]

\begin{abstract}
Los objetivos de este estudio fueron: (a) identificar los principios más importantes de un programa de formación inicial del profesorado de ciencias de secundaria; (b) identificar las estructuras de conocimiento que han construido futuros profesores de ciencias de secundaria sobre la enseñanza y el aprendizaje de las ciencias. El estudio se basa en los postulados de la cognición docente en el sentido de que los docentes construyen sus propios esquemas a partir de sus experiencias con el fin de comprender, planificar y responder a la dinámica de su aula. Este estudio cualitativo consistió en entrevistas y observaciones de cuatro futuros profesores de ciencias, y un análisis de los programas de formación inicial que han recibido. Para analizar los datos se combinaron métodos de inducción analítica de caso único y de casos cruzados. Con los datos obtenidos, se puede concluir que se adoptaron algunos de los aspectos del programa de formación inicial, tales como el aprendizaje centrado en el estudiante, el aprendizaje cooperativo, y el conocimiento pedagógico general. Sin embargo, el grado de adopción en la práctica parece estar relacionado con otras experiencias más significativas de aprendizaje de los sujetos y las limitaciones del entorno escolar.
\end{abstract}

Palabras clave: Formación profesorado de secundaria; Innovación e investigación en enseñanza de las ciencias; Conocimiento práctico profesional; Conocimiento didáctico del contenido.

The Master of Pre-service Secondary Teacher Training and the pedagogical content knowledge of the future Science, Mathematics and Technology teachers

The goals of this study were: (a) identify the most important principles of a program of pre-service secondary science teacher training, (b) identify the knowledge structures that have built future secondary science teachers about teaching and learning science. The study is based on the principles of teacher cognition in the sense that teachers build their own schedules from their experiences in order to understand, plan and respond to the dynamics of the classroom. This qualitative study consisted of interviews and observations of four pre-service science teachers, and an analysis of the initial training they have received. The analysis of the data was done by combining analytic induction methods of unique case and cross-cases. With the obtained data, it can be concluded that some aspects of the initial training program were adopted, such as student-centered learning, cooperative learning, and general pedagogical knowledge. However, the degree of adoption in practice appears to be associated with other more meaningful learning experiences of the people and the limitations of the school environment.

Keywords: Secondary teacher training; Innovation and research in science education; Pedagogical Content Knowledge.

\section{Introducción}

De acuerdo con la LOE y en línea con la adaptación de las universidades españolas al Espacio Europeo de Educación Superior, desde el curso escolar 2009-2010 las universidades han implantado un nuevo programa de formación inicial del profesorado de secundaria que tiene 
formato de Máster para post-graduados en las diferentes áreas de conocimiento incluidas en la educación secundaria obligatoria y post-obligatoria. Este Máster en Formación del Profesorado de Educación Secundaria Obligatoria, Bachillerato, Formación Profesional y Enseñanza de Idiomas (en adelante MFPS), tiene una duración de un curso académico y consta de sesenta créditos destinados a la adquisición de las habilidades necesarias para ejercer la actividad docente en secundaria. El MFPS tiene una estructura curricular común para todas las universidades del estado por orden del MEC, aunque cada universidad puede realizar pequeñas adaptaciones. Esta característica tiene la ventaja de que los resultados del análisis del desarrollo del Máster en una universidad podrían ser utilizables por otras universidades del estado.

Esta nueva iniciativa de formación inicial del profesorado de secundaria ha promovido la esperanza de obtener una mejor formación del profesorado novel para superar las dificultades actuales de la enseñanza secundaria (Vázquez et al. 2010, Oliva 2011). La preparación del profesorado se reconoce actualmente como el punto central en la reforma de la enseñanza de las ciencias (Porlán et al. 2010). Sin embargo, es necesario recordar que los programas de formación inicial del profesorado son a menudo criticados por no mirar más allá de la tarea inmediata de preparar al profesorado. Se hacen pocos intentos, por no decir ninguno, para obtener un feedback sustancial sobre su preparación, algo que sería útil para mejorar la efectividad del programa de formación inicial (Anderson y Mitchener 1994). Como resultado, los programas de formación inicial del profesorado han sido acusados de estar estancados, ser ineficaces y no responder a las necesidades cambiantes de los futuros educadores (Schnur y Golby 1995). En la actualidad, poco se sabe acerca de las relaciones entre la formación inicial que recibe el profesorado de secundaria y las habilidades que realmente adquiere para su desarrollo profesional docente (Lederman y Latz 1995).

\section{Objetivos del trabajo}

La investigación educativa ha aportado evidencias de que para enseñar contenidos no es suficiente con dominarlos sino que es necesario un conocimiento específico profesional (Shulman 1986). Estos conocimientos específicos se han denominado de diferentes formas como Conocimiento Didáctico del Contenido (CDC), saberes necesarios para la Transposición Didáctica (TD), Pedagogical Content Knowledge (PCK) o bien Conocimiento Práctico Profesional (CPP) (Bryan y Abel 1999, Wallace y Kang 2004). Dar sentido al conocimiento pedagógico en relación con la docencia de un área de conocimiento, requiere un proceso complejo de reflexión en la acción que lleva a establecer nuevas concepciones epistemológicas y un conocimiento diferenciado del pedagógico y del disciplinar para una problemática (la enseñanza de la disciplina) también diferenciada (Porlán et al. 2010). Los programas de formación del profesorado tienen como objetivo mediar en las concepciones del profesorado sobre su enseñanza y modificar su práctica hacia metodologías apoyadas por los resultados de la investigación. Es evidente que hay una necesidad de proyectos de investigación adicionales en el área de la formación inicial del profesorado y sobre su traducción en la práctica del profesorado novel. Una de las áreas más significativas para explorar estos temas tal vez sea la indicada por Finley et al. (1992), el Conocimiento Práctico Profesional (en adelante CPP) del profesorado, es decir, las estructuras de conocimiento sobre la enseñanza de la disciplina en el aula. Esta orientación hace que sea necesario el análisis de las relaciones entre los programas de formación y las estructuras de conocimiento para la enseñanza adquiridas por el profesorado novel durante el programa. Las preguntas que guiaron este estudio fueron: 
1. ¿Cuáles son las principales ideas, conceptos y creencias que desde los programas del módulo genérico, del módulo de CCNN y Matemáticas y del módulo de Tecnología del MFPS impartido en la UPV-EHU se desea que haya construido el futuro profesorado de Ciencias, Matemáticas y Tecnología como resultado de realizar el Máster?

2. ¿Cuál es la naturaleza del CPP que el profesorado novel de Ciencias, Matemáticas y Tecnología ha adquirido a lo largo del MFPS?

\section{Marco teórico}

En la actualidad el profesor no es considerado como un técnico que sigue instrucciones, sino como alguien que procesa información, toma decisiones y genera conocimiento profesional práctico. Desde la importante aportación de Shulman (1986) sobre el Conocimiento Didáctico del Contenido (Pedagogical Content Knowledge-PCK) la formación del profesorado se ha orientado hacia un mayor compromiso con el contenido concreto a enseñar. Shulman (1986) considera que el PCK incluye también las formas de representar y formular el contenido para que sea comprensible a otros. Así mismo incluye una comprensión de lo que hace sencillo o difícil el aprendizaje de temas concretos: las concepciones y preconcepciones que los estudiantes presentan cuando se enseñan estos temas. El PCK es una acumulación de diferentes elementos, tales como conocimiento de la disciplina, conocimiento de cómo aprenden los estudiantes la disciplina y de sus concepciones alternativas, conocimiento del curriculo y conocimientos generales de Pedagogía. En la medida que ha ido avanzando la investigación en formación del profesorado y en sus concepciones, estos conocimientos han ido ampliando su significado. Actualmente se habla de Conocimiento Práctico Profesional (CPP) (Porlán et al. 1996, Bradbury 2010, Bryan y Atwater 2002, Mellado et al. 2006). El CPP no es una mera aplicación de la teoría en la acción educativa, sino que como cualquier otro conocimiento profesional surge de la investigación y resolución de problemas relevantes del ámbito disciplinar o profesional (Schön 1992).

El estudio presentado en este artículo se basa en los postulados teóricos del CPP. Este estudio se refiere a la estructura curricular del MFPS de la UPV-EHU y del CPP que el profesorado ha adquirido durante el programa de formación. En este estudio al hablar de aprendizaje coincidimos con la perspectiva de Carter y Doyle (1987), quienes afirman:

Una premisa central de la ciencia cognitiva es que la comprensión es un proceso constructivo... El significado no es el resultado de la recepción o el ensayo de la información. En vez de esto, la comprensión implica una construcción activa de una representación cognitiva de los acontecimientos o conceptos y de sus relaciones en un contexto específico (p. 149).

Parece ser que una forma de investigar la estructura del conocimiento del profesorado es mediante sus recuerdos de las experiencias para construir sus constructos, un método defendido por Kelly (1955) como una forma de explorar los constructos de una persona. La teoría de Kelly de los constructos personales establece que las personas interpretan sus experiencias para producir un sistema de constructos que se utilizan posteriormente para anticipar la acción en el mundo. Las personas, ante nuevas experiencias, reinterpretarán sus experiencias para producir un sistema de constructos si el hecho de hacerlo conduce a una capacidad más sólida para anticipar la acción. Por lo tanto el profesorado recordará las experiencias del programa de formación inicial, programa que se define como cursos de metodología específica para la especialidad, cursos sobre el contenido de la especialidad y una experiencia práctica de enseñanza. Estas experiencias constituyen un mecanismo para identificar los orígenes de la cognición de los profesores noveles en relación con el programa de formación inicial del profesorado. 
Diferentes investigaciones han coincidido en definir diversas categorías del CPP (Acevedo et al. 2007, Gil et al. 1991, Jiménez Aleixandre 2010, Lederman y Latz 1995, Porlán et al. 2010, 2011, Shulman 1986). Estas categorías son: (a) el conocimiento pedagógico general, (b) conocimiento de la disciplina, (c) el conocimiento didáctico de la disciplina, y (d) conocimiento del contexto. Estas categorías ofrecen información sobre el conocimiento del docente y una síntesis fue desarrollada para los fines de este estudio. La Figura 1 proporciona el esquema de un marco para la investigación de la estructura curricular del MFPS y del conocimiento del profesorado hipotéticamente adquirido durante el programa. La estructura del CPP marco recoge cuatro áreas fundamentales (Conocimiento didáctico de la disciplina, Conocimiento de la disciplina, Conocimiento pedagógico general y Conocimiento del contexto) y diversos aspectos que recoge cada una de ellas.



Figura 1. Síntesis de diferentes propuestas sobre el modelo de CPP.

\section{Metodología}

El estudio fue de tipo cualitativo, ya que consistió en entrevistas y observación de cuatro estudiantes del MFPS pertenecientes a los módulos específicos de CCNN y Matemáticas y de Tecnología, así como el análisis de los programas del módulo genérico, del módulo de CCNN y Matemáticas y del módulo de Tecnología del MFPS de la UPV-EHU. Las entrevistas y observaciones de los cuatro estudiantes se realizaron durante las tutorías de trabajo fin de Máster (en adelante TFM) que incluyeron nueve sesiones de hora y media de duración y que se llevaron a cabo durante los meses de duración del Máster de noviembre a junio. Dos de los estudiantes fueron del curso 2010-2011 y los otros dos del curso 2011-2012.

\section{Contexto del Máster}

Los estudiantes de este estudio son licenciados en ciencias experimentales, Matemáticas e Ingeniería por la UPV-EHU. Estos estudiantes no han cursado durante la licenciatura ninguna asignatura relacionada con la enseñanza de las Ciencias y'Tecnología, ni han tenido experiencia docente en ninguna institución de enseñanza hasta llegar al Master.

El MFPS de la UPV-EHU que cursaron los estudiantes tiene una duración de un curso académico y consta de 60 créditos destinados a la adquisición por parte de los licenciados de las habilidades necesarias para ejercer la actividad docente. El currículum del Máster está regulado por la Orden Ministerial 3858/2007 de 27 de diciembre (BOE 19 de diciembre de 2007). No obstante, cada universidad puede introducir adaptaciones en la ordenación de la docencia y tipo de asignaturas concretas en los tres grandes módulos del Máster: a) Módulo genérico; b) Módulo específico; c) Practicum (prácticas y TFM). La Comisión Académica del Master de la UPV-EHU ha subdividido los módulos en materias: a) Módulo genérico (18 
créditos): Desarrollo de la Personalidad en la Adolescencia, Psicología de la Educación en Secundaria, Contextos Educativos, Orientación educativa y tutoría, La Escuela Inclusiva; b) Módulo específico (24 créditos): Complementos para la formación disciplinar. Aprendizaje y enseñanza de las materias correspondientes. Innovación docente e iniciación a la investigación educativa; c) Practicum, prácticas en centros de secundaria y TFM (12+6 créditos).

En el caso de la UPV-EHU la Comisión Académica del Máster ha considerado que integrar las prácticas en el Centro de Enseñanza con la docencia en la universidad puede ayudar a que dicha docencia se conecte de forma real con la experiencia en los centros de secundaria. Así, se han repartido las prácticas en dos períodos. El primer período se sitúa transcurridas cuatro semanas de comenzar el Máster, durante la docencia del módulo genérico. El segundo período se sitúa a las dos semanas de comenzar el módulo específico. El estudiante tiene asignados dos tutores de la universidad, uno para cada período de prácticas, más un tutor de secundaria. En el primer período la reflexión en la práctica se centra sobre problemas relacionados con la docencia del módulo genérico y por ello, el tutor/a de la universidad es un profesor/a de una asignatura del módulo genérico o bien un profesor que tiene reconocida experiencia como mentor de estudiantes en prácticas. Para el segundo período de prácticas, la reflexión en la práctica se centra en problemas relacionados con la enseñanza de la disciplina, por ello el tutor/a de la universidad es un profesor/a de una asignatura del módulo específico o bien una profesor que tiene reconocida experiencia en docencia en secundaria. Así mismo, el estudiante tiene un segundo tutor que es profesor/a del Centro docente donde realiza las prácticas. Este tutor/a es el mismo para los dos períodos de prácticas. Los tres tutores se coordinan a lo largo del Máster y la nota de prácticas es consensuada y única. Se espera que esta integración de las prácticas dentro de la docencia del Máster pueda aportar experiencias significativas de aprendizaje a los estudiantes.

En este trabajo nos vamos a referir a las especialidades de Ciencias Naturales y Matemáticas y de Tecnología del MFPS desarrollado en la UPV-EHU. La Comisión Académica, basándose en criterios profesionales del docente en la enseñanza secundaria decidió diseñar dos módulos específicos en el área de Ciencias y Tecnología. Uno de ellos es el denominado «módulo específico de CCNN y Matemáticas» que agrupa a licenciados del área de Ciencias Experimentales y Matemáticas en un solo grupo debido a que en la docencia de secundaria es frecuente que el mismo profesor/a imparta materias de ambas áreas en la enseñanza privada (educa al 49\% del alumnado en la Comunidad Autónoma Vasca) o bien, tiene la posibilidad de ocupar plaza en las dos áreas en la enseñanza pública. El módulo ofrece asignaturas obligatorias y optativas en didáctica de la Física, Química, Biología, Geología y Matemáticas, de forma que cada estudiante pueda diseñar su propio itinerario formativo de acuerdo con sus perspectivas profesionales. El otro módulo se denomina «módulo específico de Tecnología» y agrupa principalmente a ingenieros de diferentes ramas y arquitectos. En este caso se atiende a materias relacionadas principalmente con las asignaturas de Tecnología.

El número de plazas ofertadas en cada uno de los módulos descritos es de 30 estudiantes. En las tres primeras ediciones se ha completado el número de plazas y ha habido solicitudes que no se han podido atender.

\section{Los estudiantes participantes}

Los estudiantes participantes en el estudio de casos fueron seleccionados a propósito. Stake (1994) ha señalado que deben utilizarse «los casos que parecen ofrecer la oportunidad de aprender más» (p. 243). Los cuatro estudiantes participantes, identificados por sus seudónimos, Miren, Nekane, Iker y Jon, fueron seleccionados debido a que los autores del trabajo tuvieron con ellos un contacto continuado y de tutorización del TFM ( 9 sesiones de 
1,5 horas de duración a lo largo del curso). Así mismo, dentro de los estudiantes a los que tutorizamos el TFM se eligieron aquellos que ofrecían experiencias y percepciones diversas. Esta diversidad proporcionó evidencias contradictorias contra las cuales limitar, modificar o rechazar las afirmaciones que surgieron del análisis del programa del MFPS y con ello ayudar a establecer la confianza y la confirmabilidad (Guba y Lincoln 1994).

Miren es una licenciada en Física por la UPV-EHU y Máster en Física del Estado Sólido por la universidad de Utrecht. Había participado en programas de divulgación científica como monitora y posteriormente como directora del programa. Miren estaba firmemente convencida de que lo único que necesitaba era conocimientos generales de pedagogía y que tenía conocimientos suficientemente amplios de Ciencias. Durante la realización del TFM, Miren se vio confrontada con la tarea de aplicar sus creencias sobre enseñanza y aprendizaje al caso concreto de experiencias de Física para introducir conceptos de alta dificultad cognitiva a nivel de Bachillerato.

Nekane es una licenciada en Biología por la UPV-EHU. En las primeras sesiones de tutoría, Nekane indicaba claramente que el módulo genérico no le había sido útil: «No creo que me sirva para enseñar Biología...los contenidos de pedagogía me han parecido más bien consejos no apoyados en evidencias, pueden resultar o no». Sin embargo cree que necesita cursos de contenido en didáctica de Física y Matemáticas.

Iker es un ingeniero industrial de la UPV-EHU. Durante las entrevistas de tutorización expresó su convicción de que la mayoría de las materias del módulo genérico y del módulo específico le habían resultado útiles para enseñar, a diferencia de las opiniones de Miren y Nekane. Por ejemplo, cuando se le pidió que explicara su motivación para el uso de grupos de cooperación, mencionó sus experiencias en el módulo específico y su experiencia en el TFM.

Jon es un licenciado en Matemáticas por la UPV-EHU. Ha sido elegido por tener una licenciatura diferente a la de los otros tres. Reconoce su poco conocimiento del área de ciencias experimentales, pero no cree útil su formación en dicha área porque piensa que su futuro estará en la enseñanza de las Matemáticas. No obstante piensa que en general los módulos del Máster le serán útiles.

Cada una de estas personas, si las observamos superficialmente, tiene percepciones diferentes del programa de formación inicial del profesorado. Las cuatro fueron elegidas intencionalmente por este motivo. El estudio de casos observa por debajo de la superficie con el fin de extraer las experiencias fundamentales de las cuales los participantes tal vez no sean conscientes, según lo sugerido por Kelly (1995). El análisis cruzado de casos buscó temas comunes entre estas personas dispares.

\section{El programa de las materias del módulo genérico y de los módulos de CCNN y matemáticas y Tecnología}

Los programas del MFPS elegidos son los de las seis materias de tres créditos del módulo genérico (desarrollo de la personalidad en la adolescencia, psicología de la educación en secundaria, contextos educativos, orientación educativa y tutoría, sistema escolar, familia y derechos humanos en una sociedad en cambio y la escuela inclusiva), las once materias de tres créditos del módulo de CCNN y Matemáticas (cinco materias obligatorias y tres optativas a elegir de entre seis) y las ocho materias obligatorias de tres créditos del módulo de Tecnología.

\section{Análisis de datos}

Se utilizaron métodos de inducción analítica de caso único y casos cruzados (Patton 1990) para analizar los datos. El análisis de los programas del MFPS se realizó mediante una 'matriz 
de análisis de programas' construida a partir de los elementos de CPP de la figura 1. Se construyeron registros de caso para cada estudiante del MFPS a partir de las fuentes de datos identificadas en la tabla 1, que muestra la relación de los datos con las preguntas de investigación de este estudio. Las entrevistas y datos de las tutorías se registraron mediante notas que luego se convirtieron en registros del caso (Stake 1994) que fueron analizadas mediante criterios de modalidades de enseñanza de acuerdo con Furió y Carnicer (2002, véase los cuadros III, p. 53, y IV, p. 54).

Tabla 1. Fuentes de datos según las preguntas de investigación.

\begin{tabular}{|l|c|c|}
\hline Fuente & Pregunta de investigación 1 & Pregunta de investigación 2 \\
\hline Análisis de los programas de: & & \\
6 materias del módulo genérico & $\mathrm{X}$ & \\
11 materias del módulo de CCNN y Matemáticas & $\mathrm{X}$ & \\
8 materias del módulo de Tecnología & $\mathrm{X}$ & $\mathrm{X}$ \\
\hline Estudiantes del Máster & & $\mathrm{X}$ \\
Notas durante la tutoría & & $\mathrm{X}$ \\
Entrevista post-Máster & & \\
Análisis del TFM & & \\
\hline
\end{tabular}

En las sesiones de tutoría se tomaron notas de cada caso y se codificaron para triangular con el análisis del TFM y buscar evidencias contradictorias.

Este trabajo tiene limitaciones que es necesario comentar antes de presentar los resultados obtenidos. La metodología del estudio de casos puede no ser generalizable excepto cuando otro lector/investigador vea reflejado el caso en su práctica. Los resultados no son fáciles de replicar, ya que la elección de los casos puede ser selectiva, parcial, personal y/o subjetiva. Así mismo, estos estudios pueden tener problemas de sesgo del observador, a pesar de los intentos de abordarlos desde una metodología reflexiva (Cohen et al. 2007). Una última limitación es el contexto y cultura propia de la organización del programa de la UPV-EHU. En efecto, el contexto puede determinar el cómo y el qué de la enseñanza y el aprendizaje. Sin embargo, la metodología de casos aporta datos cualitativos y particulares que pueden ser no tenidos en cuenta en estudios con diseños cuantitativos. Además, los datos cualitativos aportados pueden ser el punto de partida de un estudio más amplio con diseños cuantitativos para responder con mayor número de participantes a las preguntas de investigación planteadas.

\section{Resultados y discusión}

El proceso de análisis inductivo empleado permitió enmarcar los resultados de los estudios analíticos de programas y trabajos fin de Máster cruzados como afirmaciones empíricas de las entrevistas, considerando los datos como garantías probatorias (Erickson 1986). La discusión que se presenta aquí se centra en los hallazgos cruzados emergentes.

\section{Conocimiento pedagógico general}

Basándose en los datos de los programas de MFPS, puede concluirse que el programa de las diferentes materias pone el énfasis en las características del modelo de conocimiento práctico del profesorado (véase la figura 1): a) conocimiento pedagógico general; b) Conocimiento del contexto. Los programas de las asignaturas mencionan contenidos concretos de ambas áreas de conocimiento y presentan las teorías educativas relevantes. 
Los programas de las materias están principalmente centrados en la disciplina y no mencionan contenidos transversales que relacionen las diversas materias. No aparecen datos que evidencien que las teorías impartidas se apliquen a problemas o casos concretos en el aula de secundaria. No parece que, en general, los programas estén centrados en los futuros docentes de secundaria. Los estudiantes participantes consideran que los contenidos de estas áreas educativas son interesantes como información pero tienen dudas de su utilidad dentro de su profesión como profesor de ciencias. Miren explica:

"He recibido mucha información sobre cómo aprenden los estudiantes de secundaria, sobre el contexto educativo y sobre cómo llevar una tutoría con adolescentes. Uno puede pensar sobre esto y escribir un informe. Mi pregunta es cómo adaptar todo esto en mi clase de ciencias. No estoy segura de que toda esta teoría la pueda utilizar para enseñar mejor ciencias."

Jon hace referencia a una cuestión que ha sido señalada como problema por los cuatro estudiantes:

\footnotetext{
"El módulo de pedagogía me ha permitido conocer el contexto de la educación secundaria, con aspectos que yo como alumno no me daba cuenta o me pasaron inadvertidos. Sin embargo, he echado en falta el análisis de casos concretos que pueden sucederme cuando empiece a dar clases. En las prácticas me ha tocado un centro subvencionado [no se va a citar su nombre ni ubicación] en el que la profesora tenía graves problemas de disciplina en el aula. Me ha alarmado el comportamiento de algunos estudiantes de $3^{\circ}$ y $4^{\circ}$ de ESO, ¿con qué teoría pedagógica se afrontan estos problemas? Sobre esto no hemos comentado nada en el módulo pedagógico y sólo en una materia de didáctica específica se ba comentado lo importante que es interesar e implicar al alumnado."
}

Una de las preocupaciones más comentadas por los cuatro estudiantes es el problema de la disciplina. Se trata de una de las preocupaciones citadas desde hace décadas por el profesorado novel a nivel internacional (Adams y Krockover 1997). Los cuatro estudiantes tuvieron dificultades para mencionar información de las materias del módulo genérico que hubieran podido aplicarse dentro de su clase de ciencias para controlar el aula. Sin embargo, sí indicaron acciones concretas relacionadas con el módulo genérico en relación a cómo establecer relaciones con los otros profesores y la organización escolar en el centro. Nekane explica:

"El módulo genérico me informó sobre las diferentes facetas como profesora de secundaria. No se trata de entrar en tu aula y explicar Biología. En las prácticas pude ver los diferentes contextos en los que nos tenemos que mover: profe, tutor, gestos, puente con la familia, etc. Esta parte de la profesión si fue nueva para mi. Sin embargo, he recibido la información en «cajas», no sé si tengo una visión global de lo que me espera como profesora."

\section{Conocimiento didáctico de la disciplina}

Los programas de los módulos específicos de CCNN y Matemáticas, y Tecnología cubren ampliamente los contenidos de CPP de la figura 1: a) estrategias de instrucción y evaluación; b) conocimiento teórico y epistemológico de la disciplina. En relación a los programas de CCNN y Matemáticas se observa un enfoque centrado en las concepciones alternativas de los estudiantes y en las estrategias de enseñanza para el diseño de unidades didácticas. No obstante, al igual que en el módulo genérico, no hay evidencias de que se traten temas transversales que se compartan o relacionen las diferentes materias del módulo. Miren expresa que:

"A la hora de hacer la unidad didáctica que propongo en el TFM, me he encontrado un poco confusa pues en diferentes materias hemos visto estrategias de instrucción a veces diferentes. En todas las materias se daba importancia a las concepciones alternativas de los estudiantes, pero luego no queda claro cuál debe ser la estrategia de enseñanza a seguir."

Además, nótese cómo Miren echa en falta una «estrategia de enseñanza a seguir», esto es, demanda de sus profesores «la fórmula» que resuelva el problema. Volveremos sobre esta demanda de los estudiantes en el próximo apartado.

Sin embargo, las materias del módulo de Tecnología sí indican explícitamente temas transversales a tratar en diversas asignaturas. En concreto, en la materia de «innovación educativa en tecnología» el programa menciona cómo utilizar conocimientos impartidos en el 
programa de otras tres materias. Iker expresa su convicción de que sus experiencias en dicha asignatura fueron útiles para construir su TFM:

"En la asignatura de «innovación» hemos construido un proyecto que nos hacía integrar conocimientos de otras materias de la especialidad. Hemos tenido que discutirla con otros compañeros y presentarla. Esto me ha becho construir una serie de actividades a trabajar con los estudiantes, que me han sido muy útiles para el TFM."

Además, Iker parece haber interiorizado la necesidad de incluir como objetivos de enseñanza aspectos epistemológicos de la Ciencia, cuando afirma:

"La competencia en el conocimiento y la interacción con el mundo físico también requieren conocer de qué manera se genera el conocimiento científico. Para lo que es necesaria la familiarización con el método de trabajo científico. Esta familiarización puede realizarse desde la discusión sobre el interés de las situaciones propuestas, de manera que se facilite la comprensión de dichas situaciones, hasta la propuesta de hipótesis y relaciones fundamentadas que lleven a la elaboración de conclusiones."

La mayor interrelación en el programa del módulo de Tecnología puede ser debido a que hay un reducido número de profesores que se coordinan entre sí al enseñar sus materias. El programa del módulo ha sido discutido en grupo a lo largo de los tres años de experiencia del MFPS y se han realizado las modificaciones pertinentes. En el caso del módulo de CCNN y Matemáticas esto no ha sucedido.

\section{Conocimientos del diseño de unidades didácticas y la evaluación}

Los futuros profesores sienten la necesidad de aprender sobre la organización básica de una unidad didáctica y la forma de utilizar diversas fuentes para desarrollar las estrategias instructivas (Furió y Carnicer 2002). Esto puede traducirse, en ocasiones, en la petición de que se les proporcione de forma rápida una serie de «fórmulas» para aplicar en la enseñanza. Esto coincide con la afirmación de Rodríguez (1993) cuando indica que algunos estudiantes buscan el falso concepto de «aprender una serie de trucos que se pueden extrapolar desde el contexto académico al contexto escolar» (p.219). Los cuatro estudiantes participantes expresan su convicción de que las materias del módulo les han servido para aprender organizar una unidad didáctica, aunque cada uno con sus propias salvedades. Los estudiantes de CCNN y Matemáticas, Miren, Nekane y Jon hacen muchos comentarios sobre tratamiento de concepciones alternativas, pero expresan sus dudas sobre cómo evaluar. Nekane indica:

\footnotetext{
"He organizado la unidad didáctica del TFM desde las concepciones alternativas de los estudiantes y sus intereses, haciendo un tratamiento en grupo de problemas que yo he ido proponiendo para que lleguen a comprender los conceptos de la lección. Sin embargo, no he aprendido mucho sobre la calificación y cómo manejar las posibles quejas."
}

Iker, del módulo de Tecnología, se siente con capacidad de aplicar dos aspectos que ha trabajado en varias materias del módulo: la evaluación y el trabajo cooperativo en el aula. Iker expresa en la tutoría del TFM:

"Aprendí sobre cómo organizar a los estudiantes para que trabajen en grupo y de forma cooperativa. Por eso he puesto muchas actividades de este tipo en el TFM. Luego lo evalúo con una serie de rúbricas que me han dado en dos de las materias del módulo."

Tras su experiencia en el Prácticum, Iker también es capaz de criticar los programas y objetivos de Formación Profesional:

\footnotetext{
"Los alumnos de formación profesional tienen el riesgo de convertirse en meras herramientas de la industria, si sólo se actúa en relación a los objetivos de las empresas sin tenerse en cuenta la importancia que tienen estos estudios en el crecimiento personal de estos alumnos. Hoy en día se les está enseñando a hacer de una forma programada y memorística dirigida a las tareas que pronto desempeñarán en la industria."
}

\section{Decisiones sobre estrategias instructivas}

Analizando las entrevistas en tutoría y el TFM, de acuerdo con los cuadros III y IV de Carnicer y Furió (2002) que proporcionan una escala de mayor proximidad o no a estrategias 
instructivas de tipo constructivista; Nekane e Iker fueron clasificados como 'profesores constructivista iniciales'. El profesorado constructivista, según los cuadros de Carnicer y Furió (2002), es el que realiza acciones tales como (a) discutir del interés del tema con los estudiantes; (b) negociar de la comprensión de ideas clave con los estudiantes; (c) proponer problemas y proyectos ««abiertos» a concretar con el estudiante; (d) conducir a los estudiantes a reconstruir cómo se ha utilizado la evidencia para formular ideas científicas; (e) utilizar métodos centrados en el estudiante tales como el trabajo en grupo, los mapas de conceptos y la escritura para representar ideas; y (f) utilizar diferentes formas de evaluación que se integran con la instrucción. En cambio, Miren y Jon fueron clasificados como 'profesores centrados en la disciplina'. Este profesorado realiza acciones tales como (a) un uso predominante de los métodos de enseñanza centrados en el profesor; (b) resolución de problemas «con receta» cuyas respuestas son conocidas; (c) instrucción que busca corregir ideas no científicas sin consideración del conocimiento previo de los estudiantes; (d) escritura para reconfigurar la información proporcionada, y (e) uso limitado de las evaluaciones alternativas.

La diferencia clave entre las dos clasificaciones no reside en el tipo de actividad utilizada, ya que ambos grupos propusieron a menudo actividades similares en el TFM, sino en la participación de los estudiantes y en la secuencia posterior de instrucción y evaluación. Nekane e Iker integraron más la instrucción y la evaluación que Miren y Jon, quienes tendieron a apoyarse más en exámenes finales que en problemas abiertos y en proyectos realizados por los estudiantes.

Los cuatro estudiantes admiten que las diferentes materias del MFPS les han ayudado en el TFM y reconocen que la información recibida les ayudó a examinar sus puntos de vista sobre la enseñanza de su materia y a articular el proyecto del TFM. Sin embargo, en las entrevistas surgen con frecuencia sus experiencias como estudiantes. No debería extrañar que la larga experiencia como estudiantes en secundaria y en la Universidad de los cuatro futuros profesores sea parte importante de sus decisiones en relación a la enseñanza de la disciplina (Bryan y Abell 1999). La teoría de Kelly (1955) de principios personales sugiere que las decisiones sobre la enseñanza incorporan experiencias anteriores en Secundaria y Universidad. Jon con frecuencia toma como modelo las acciones de su profesor de Matemáticas en el instituto. Según comentó:

"Mi profesor de Matemáticas de bacbillerato fue realmente la fuente de inspiración para proponer actividades de enseñanza y de relación con el grupo de estudiantes en el aula. Él es una de las razones para que quiera ser profesor de Matemáticas. Era un profesor que explicaba muy bien, con un discurso pausado y coherente. Te hacia ver los conceptos."

Jon toma también como referencia válida el ejemplo de su profesor mentor de prácticas:

"El profesor junto al que he realizado las prácticas disponía de gran destreza profesional en el desarrollo de su actividad docente y además de dominar la materia impartida, explicaba la teoría y la resolución de ejercicios de manera excepcional, fomentando en todo momento el interés del alumnado bacia la materia."

Luego constata el fracaso de la enseñanza de este profesor-modelo, pero en ningún momento se muestra crítico con él, más bien atribuye el fracaso a los estudiantes:

"A pesar de ello, pude evidenciar en el alumnado una falta evidente de implicación, concentración e interés tanto a la hora de atender a las explicaciones del profesor, como a la de realizar los ejercicios. No tendían a reflexionar ni relacionar los ejercicios que se les planteaban con la teoría anteriormente explicada."

Así pues, a pesar de la formación recibida y la experiencia proporcionada a lo largo del TFM, parece que Jon propone un modelo similar al de su profesor de instituto (expositivo) en el desarrollo de sus actividades en el TFM.

Nekane usa sus experiencias como profesora de «clases particulares» ayudando a estudiantes fuera de la enseñanza reglada, para apoyar sus decisiones sobre la instrucción en el desarrollo de actividades del TFM. Ella reconoce esta experiencia como significativa a la hora de 
reflexionar acerca de su propia concepción sobre la enseñanza. Esto parece haberse reflejado en la forma en que ella organiza las actividades del TFM. En las actividades que propone no se prioriza sólo el conocimiento de la materia en sí, ya que hace uso de la discusión y la demostración mediante evidencias (Jiménez Aleixandre 2008).

Al comenzar el TFM los cuatro estudiantes se apoyaron, sin tener en cuenta el conocimiento impartido en los módulos del MFPS, en (a) los libros de texto del instituto donde realizaron las prácticas y (b) los cursos de la facultad.

\section{Conclusiones}

En este estudio hemos realizado un análisis de los programas de las materias que componen el módulo genérico y los módulos específicos de CCNN y Matemáticas y Tecnología de la UPVEHU. El resultado del análisis de acuerdo con el modelo de CPP definido, nos indica que los programas cubren los contenidos de CPP y que, en el caso del módulo genérico, están centrados en la disciplina más que en los problemas de enseñanza que la bibliografía señala para el profesorado de Secundaria. Para el caso de los módulos específicos, se identificaron evidencias de que los programas se centran más en la problemática del profesorado de secundaria. En el caso de módulo de Tecnología se han identificado contenidos transversales que afectan a varias materias y que proponen la utilización de proyectos con contenidos de varias materias. En general, se puede concluir que se imparten contenidos de acuerdo con los resultados de la investigación educativa, aunque en el caso del módulo genérico la enseñanza parece centrada en la transmisión de la información más que en compartir y analizar problemas con los estudiantes. Uno de los factores que puede contribuir a esta metodología expositiva puede ser el elevado número de estudiantes en el módulo genérico (más de 70 estudiantes en alguno de los tres Campus). Por el contrario el grupo se reparte en un número máximo de 30 estudiantes en los diferentes módulos específicos.

En relación al estudio de casos con cuatro estudiantes de los módulos de CCNN y Matemáticas y Tecnología, una tendencia que se aprecia en los datos es la utilización de las ideas presentadas en los módulos del MFPS dentro de la estructura de conocimiento que expresan en las entrevistas y muestran en su TFM. A pesar de que en los programas de los módulos específicos se aprecia el uso de metodologías constructivistas, parece que muchas de las concepciones previas de los cuatro estudiantes sobre la enseñanza de las ciencias todavía se mantienen o han sido sólo ligeramente modificadas. Creemos que esto podría ser debido a tres causas. Primero, el cambio requiere experiencias que revelen los beneficios de la metodología constructivista (Scott et al. 2008). Esto podría justificar que Nekane e Iker adoptaran métodos constructivistas que fueron propugnados en el módulo específico, ya que tenían experiencias previas que les hacía intuir los beneficios de las actividades centradas en el estudiante. Una segunda causa puede ser el hecho de que prácticamente toda su experiencia de enseñanza en la licenciatura les proporcionó un gran número de contra-modelos en relación al enfoque constructivista de la enseñanza propugnado en los módulos del MFPS. No se pueden despreciar las experiencias de los estudiantes y su identificación con determinados profesores de secundaria y universidad que son contra-modelos de una enseñanza centrada en la compresión. Por último, si las concepciones previas de enseñanza y aprendizaje están profundamente arraigadas, el cambio didáctico puede tardar años en producirse o no llegar nunca, como quizá sea el caso de Miren y Jon; Nekane e Iker parecen tener concepciones y experiencias previas más cercanas al modelo propuesto en los módulos específicos del MFPS.

Estas reflexiones sugieren dos vías de actuación. La primera de ellas es que la forma en que se imparte el MFPS debería cambiar. Debería hacer más uso de estrategias de enseñanza que analizaran «escenarios» reales de problemas en la enseñanza secundaria. Estas estrategias 
podrían incidir en mayor medida sobre las concepciones previas de los estudiantes acerca de la enseñanza, informando acerca de la realidad de la práctica del futuro profesorado, reforzando así las nuevas orientaciones del curriculum de Secundaria. La segunda vía es comenzar una reflexión crítica sobre la enseñanza en las titulaciones universitarias y su adecuación a los resultados que aporta la investigación educativa (Mellado 1999). Gran parte de la enseñanza universitaria se preocupa, siguiendo los parámetros propios - pero ya en crisis- de las sociedades industriales, de transmitir la información. En los estudios directamente encaminados a fines profesionales, y más aún en los grados, se observan graves ausencias en aspectos tan importantes como implicar a los estudiantes en un proceso de enfrentarse con la incertidumbre, con la profundización en los significados y con el contexto. No obstante, este patrón transmisivo, probablemente muy unido al notable crecimiento de las universidades desde hace tres décadas, convive con otra idea que ha caracterizado a las universidades desde su nacimiento: hacer pensar a los estudiantes (Boulton y Lucas 2008).

Este estudio tiene un carácter exploratorio con las limitaciones ya comentadas en la sección sobre metodología. No obstante tiene también implicaciones para estudios futuros y experimentos potenciales. Por ejemplo: ¿cuál es la naturaleza de las experiencias de enseñanza dentro del programa de MFPS que han influido en los futuros docentes de Ciencias, Matemáticas y Tecnología para construir su comprensión de la enseñanza y el aprendizaje según los mismos principios del programa? ¿Esta influencia depende únicamente de cada miembro individual del cuerpo de profesores universitarios o puede repetirse en otras instituciones? ¿Cuáles son las estructuras subyacentes de la enseñanza universitaria en el grado o licenciatura? Apenas existen estudios que analizan la estructura de la enseñanza universitaria y el pensamiento de su profesorado. Se necesitan más estudios para abordar estas y otras preguntas (Bryan y Atwater 2002). Lo que resulta evidente es que en los resultados obtenidos subyace una necesidad de cambio educativo. Los profesores y profesoras de Secundaria y de Universidad suelen ser, en su mayoría, buenos docentes dentro de los parámetros de una sociedad en la que los procesos de producción y transmisión del conocimiento eran lineales, tenían un ritmo lento y estaban estrechamente vinculados a un espacio concreto. Una de las dificultades para el cambio educativo es el conflicto que una parte importante del profesorado vive entre su deseo de promover el desarrollo de los estudiantes mediante su participación en el proceso educativo, y las concepciones implícitas sobre educación definidas social y culturalmente.

\section{Referencias bibliográficas}

Acevedo-Díaz J. A., Vázquez-Alonso A., Manassero-Mas Maa, M., Acevedo-Romero P. (2007) Consensos sobre la Naturaleza de la Ciencia: fundamentos de una investigación empírica. Revista Eureka sobre Enseñanza y Divulgación de las Ciencias 4(1), 42-66.

Adams P. E., Krockover G. H. (1997) Concerns and perceptions of beginning secondary science and mathematics teachers. Science Education 81, 29-50.

Anderson R. D., Mitchener C. P. (1994) Research on science teacher education, pp. 3-44 en D. L. Gabel (Ed.), Handbook of research on science teaching and learning. New York. MacMillan.

Boulton G., Lucas C. (2008) What are universities for? Leuven. League of European Research Universities (LERU).

Bradbury L. U. (2010) Educative Mentoring: Promoting reform-based science teaching through mentoring relationships. Science Education 94, 1049-1071. 
Bryan L. A., Abell S. K. (1999) The development of professional knowledge in learning to teach science. Journal of Research in Science Teaching 36, 121-139.

Bryan L. A., Atwater, M. M. (2002). Teacher beliefs and cultural models: A challenge for science teacher preparation programs. Science Education 86, 821-839.

Carter K., Doyle W. (1987) Teacher's knowledge structures and comprehension processes, pp. 147-160 en J. Calderhead (Ed.), Exploring teachers thinking. London. Cassell.

Cohen L., Manion L., Morrison K. (2007) Research methods in Education. Capítulo 11, p. 253. London. Routledge.

Erickson F. (1986) Qualitative methods in research on teaching, pp. 119-161 en M. C. Wittrock (Ed.), Handbook of research on teaching. New York. MacMillan.

Finley F., Lawrenz F., Heller P. (1992) A summary of research in science education. Science Education 76, 239-254.

Furió C., Carnicer J. (2002) El desarrollo profesional del profesor de ciencias mediante tutorías de grupos cooperativos. Estudio de ocho casos. Enseñanza de las Ciencias 20(1), 47-73.

Gil D., Carrascosa J., Furió C., Martínez-Torregrosa J. (1991) La enseñanza de las ciencias en la educación secundaria.. Barcelona. Horsori/ICE.

Guba E. G., Lincoln Y. S. (1994) Competing paradigms in qualitative research, pp. 105-117 en N. K. Denzin e Y. S. Lincoln (Eds.), Handbook of qualitative research. Thousand Oaks, CA. Sage.

Jiménez Aleixandre M. P. (2008) Designing argumentation learning environments, pp. 91-115 en S. Erdurán y M. P. Jiménez Aleixandre (Eds.) Argumentation in science education: Perspectives from classroom-based research. Dordrecht. Springer.

Jiménez Aleixandre M. P. (2010) 10 Ideas Clave. Competencias en argumentación y uso de pruebas. Barcelona. Graó.

Kelly G. A. (1995) The psychology of personal constructs. New York. Norton.

Lederman N. G., Latz M. S. (1995) Knowledge structures in the preservice science teacher: Sources, development, interactions, and relationships to teaching. Journal of Science Teacher Education 6(1), 1-19.

Mellado V. (1999) La formación didáctica del profesorado universitario de ciencias. Revista interuniversitaria de formación del profesorado 34(1), 231-241.

Mellado V., Ruiz C., Bermejo M. L., Jiménez R. (2006) Contributions from the philosophy of science to the education of science teachers. Science and Education 15(5), 419-445.

Oliva J. Ma . (2011) Dificultades para la implicación del profesorado de Educación Secundaria en la lectura, innovación e investigación en didáctica de las ciencias (I): el problema de la inmersión. Revista Eureka sobre Enseñanza y Divulgación de las Ciencias 8(1), 41-53.

Patton M. Q. (1990) Qualitative evaluation and research methods, 2nd ed. Newbury Park, CA. Sage.

Porlán R., Martín del Pozo R., Ribero A., Harres J., Azcárate P., Pizzato M. (2010) El cambio del profesorado de ciencias I: Marco teórico y formativo. Enseñanza de las Ciencias 28(1), $31-46$.

Porlán, R., Martín del Pozo, R., Ribero, A., Harres, J., Azcárate, P. y Pizzato M. (2011) El cambio del profesorado de ciencias II. Enseñanza de las Ciencias 29(3), 31-46. 
Rodríguez A. J. (1993) A dose of reality: Understanding the origin of the theory/practice dichotomy in teacher education from the students' point of view. Journal of Teacher Education 44, 213-222.

Schnur J. O., Golby M. J. (1995) Teacher education: A university mission. Journal of Teacher Education 46, 11-18.

Schön D. (1992) La formación de profesionales reflexivos. Hacia un nuevo diseño de la enseñanza y el aprendizaje en las profesiones. Barcelona. Paidós.

Scott P., Asoko H., Leach J. (2008) Student conceptions and conceptual learning in science. pp. 31-52 en A. K. Abell y N. G. Lederman, Handbook of research on science education. New York. Routledge.

Shulman L. (1986) Knowledge and teaching: Foundations of the new reform. Harvard Educational Review 57(1), 1-22.

Stake R. E. (1994) Case studies, pp. 236-247 en N. K. Denizen e Y. S. Lincoln (Eds.), Handbook of qualitative research. Thousand Oaks, CA. Sage.

Vázquez J. M., Jiménez R., Mellado V. (2010) Los obstáculos para el desarrollo profesional de una profesora de enseñanza secundaria en ciencias experimentales. Enseñanza de las Ciencias 28(3), 417-432.

Wallace C. S., Kang N. (2004) An investigation of experienced secondary science teachers'beliefs about inquiry: an examination of competing belief sets. Journal of Research in Science Teaching 41(9), 936-960. 